Georgian Mathematical Journal

Volume 8 (2001), Number 1, 61-67

\title{
PERTURBATION OF A FREDHOLM COMPLEX BY INESSENTIAL OPERATORS
}

\author{
JIM GLEASON
}

\begin{abstract}
The work of Ambrozie and Vasilescu on perturbations of Fredholm complexes is generalized by discussing the stability theory of Banach space complexes under inessential perturbations.

2000 Mathematics Subject Classification: Primary: 47A53; Secondary: $47 \mathrm{~A} 13$.

Key words and phrases: Fredholm, Banach space complex, inessential operators.
\end{abstract}

\section{INTRODUCTION}

The aim of this paper is to show that a Banach space complex over the complex numbers which is a perturbation of a Fredholm complex by inessential operators is also Fredholm. This problem arises naturally as an extension of the problem of finding the largest ideal $P$ such that the Fredholmness of an operator is stable under perturbations by elements of $P$. This ideal is the ideal of inessential operators [1].

Definition 1. An operator $S \in B(E, F)$ belongs to the ideal of inessential operators, $P(E, F)$, if for each $L \in B(F, E)$ there exist $U \in B(E, E)$ and $X \in K(E, E)$ such that

$$
U\left(I_{E}-L S\right)=I_{E}-X
$$

Remark: If we let $K(E, F)$ be the ideal of compact operators from $E$ to $F$, then $K(E, F) \subseteq P(E, F)$ with strict inclusion in general with an example given in [5] using the space $E=l^{q} \times L^{p}$, where $L^{p}=L^{p}(-1,1)$ and $1<p<q<2$. However, in the case where $H$ is a Hilbert space, by looking at the real and imaginary parts of an inessential operator, we find that $K(H)=P(H)$. In the following, we will show that these results can be extended to the realm of Banach space complexes.

Notation: A Banach space complex is a sequence $(X, \delta)=\left(X^{p}, \delta^{p}\right)_{p \in \mathbb{Z}}$, where $X=\left(X^{p}\right)_{p \in \mathbb{Z}}$ are Banach spaces, and $\delta=\left(\delta^{p}\right)_{p \in \mathbb{Z}}$ are continuous linear maps such that $\delta^{p}: X^{p} \rightarrow X^{p+1}$ and $\delta^{p+1} \delta^{p}=0$ for all $p \in \mathbb{Z}$ (in other words, $R\left(\delta^{p}\right)$ is contained in $N\left(\delta^{p+1}\right)$ where $R(T)$ and $N(T)$ are the image of $T$ and the kernel of $T$ respectively).

ISSN 1072-947X / \$8.00 / C) Heldermann Verlag www.heldermann.de 
Since the domain of definition $X^{p}$ of $\delta^{p}$ is uniquely determined by $\delta^{p}$, we will usually identify the complex $(X, \delta)$ with the sequence $\delta$, which is also called a complex.

We will also identify the Banach spaces $X^{p}$ with closed subspaces of

$$
\mathcal{X}:=\left\{\left(x_{p}\right)_{p \in \mathbb{Z}} \in \prod_{p \in \mathbb{Z}} X^{p}: \sum_{p \in \mathbb{Z}}\left\|x_{p}\right\|^{2}<\infty\right\}
$$

Then a complex $(X, \delta)$ as above will be called a complex in $\mathcal{X}$ and the class of complexes in $\mathcal{X}$ will be denoted by $\partial(\mathcal{X})$.

Let $\delta=\left(\delta^{p}\right)_{p \in \mathbb{Z}}$ be a complex. The homology, $H(\delta)$, of $\delta$ is the sequence of linear spaces $\left(H^{p}(\delta)\right)_{p \in \mathbb{Z}}$, where

$$
H^{p}(\delta):=N\left(\delta^{p}\right) / R\left(\delta^{p-1}\right), \quad p \in \mathbb{Z} .
$$

Let $\delta=\left(\delta^{p}\right)_{p \in \mathbb{Z}}$ be a complex. We say that $\delta$ is Fredholm if $\operatorname{dim} H^{p}(\delta)<\infty$ for all $p \in \mathbb{Z}$ and $\operatorname{dim} H^{p}(\delta)=0$ for all except a finite number of indices. For a Fredholm complex $\delta$, we define the index of $\delta$ by the formula

$$
\text { ind } \delta:=\sum_{p \in Z}(-1)^{p} \operatorname{dim} H^{p}(\delta)
$$

In order to check that this is a generalization of Fredholm operators, let $\delta^{0}: X^{0} \rightarrow X^{1}$ be a bounded linear operator, and let $\delta$ be the complex associated with $\delta^{0}$. Note that $\delta$ is Fredholm if and only if $\operatorname{dim} N\left(\delta^{0}\right)<\infty$ and $\operatorname{dim} X^{1} / R\left(\delta^{0}\right)<\infty$. Thus, $\delta^{0}$ is Fredholm in the usual context if and only if $\delta$ is Fredholm and $R\left(\delta^{0}\right)$ is closed (which in fact follows from the property that $\left.\operatorname{dim} X^{1} / R\left(\delta^{0}\right)<\infty\right)[1$, p. 50].

Much of the following is based on the book by Ambrozie and Vasilescu [1] where the authors prove the following result.

Theorem 1 ([1, II.3.22]). Let

$$
0 \rightarrow X^{0} \stackrel{\alpha^{0}}{\longrightarrow} X^{1} \stackrel{\alpha^{1}}{\longrightarrow} \cdots \stackrel{\alpha^{n-1}}{\longrightarrow} X^{n} \rightarrow 0
$$

be a Fredholm complex of Banach spaces and continuous operators. If

$$
0 \rightarrow X^{0} \stackrel{\tilde{\alpha}^{0}}{\longrightarrow} X^{1} \stackrel{\tilde{\alpha}^{1}}{\longrightarrow} \cdots \stackrel{\tilde{\alpha}^{n-1}}{\longrightarrow} X^{n} \rightarrow 0
$$

is another complex such that $\alpha^{p}-\tilde{\alpha}^{p} \in K\left(X^{p}, X^{p+1}\right)$ for all $p=0,1, \ldots, n$, then the latter complex is also Fredholm.

In this paper, we wish to generalize this theorem from a perturbation in the ideal of compact operators to the ideal of inessential operators. 


\section{MAin Results}

Following the ideas in [1] and [2], we will work with a class of operators larger than $B(Z, X)$. These will be the homogeneous operators which we will denote by $H(Z, X)$. An operator ${ }^{1} T: Z \rightarrow X$ is called homogeneous if $T(\lambda x)=\lambda T(x)$ for all complex numbers $\lambda$ and all $x \in X$. A homogeneous operator $\phi \in H(Z, X)$ is called compact if $\phi(A)$ is relatively compact in $X$ for every bounded $A \subset Z$. The compact homogeneous operators will be denoted by $K_{h}(Z, X)$. Furthermore, we have the following extension of the inessential operators:

Definition 2. An operator $\theta \in H(E, F)$ belongs to the ideal of homogeneous inessential operators, $P_{h}(E, F)$, if for each $L \in H(F, E)$ there exist $U \in H(E, E)$ and $X \in K_{h}(E, E)$ such that

$$
\left(I_{E}-\theta L\right) U=I_{E}-X .
$$

Clearly we have that $K_{h}(Z, X) \supset K(Z, X)$ and $P_{h}(E, F) \supset P(E, F)$.

In order to complete the desired proofs, the following lemma regarding homogeneous inessential operators and their invariant subspaces.

Lemma 2. If $\theta \in P_{h}(X)$ and if $Y$ is a closed linear subspace of $X$ such that $\theta Y \subset Y$ then $\theta$ restricted to $Y$ is a homogeneous inessential operator on $Y$.

Proof. Let $L \in H(Y)$. By Theorem I.5.9 and Lemma I.5.8 in [1], we know that there exists a homogeneous projection $P \in H(X, Y)$ of $X$ onto $Y$ such that

$$
P(x+y)=P(x)+y, \quad x \in X, y \in Y .
$$

If we define $\widetilde{L}=L P$, then $\left.\widetilde{L}\right|_{Y}=L$. Since $\theta \in P_{h}(X)$, we have that there exists a $U \in H(X)$ and a $K \in K_{h}(X)$ such that

$$
\left(I_{X}-\theta \widetilde{L}\right) U=I_{X}-K .
$$

Thus

$$
\left.\left(I_{Y}-\left.\theta\right|_{Y} L\right) P U\right|_{Y}=I_{Y}-\left.P K\right|_{Y}
$$

Therefore, $\left.\theta\right|_{Y}$ is inessential on $Y$.

Definition 3. We say a complex $\alpha=\left(\alpha^{p}\right)_{p \in \mathbb{Z}}$ with $\alpha^{p} \in B\left(X^{p}, X^{p+1}\right)$ for all $p \in \mathbb{Z}$ has a homogeneous splitting if there exists a collection of operators $\theta\left(\theta^{p}\right)_{p \in \mathbb{Z}}$ and $\nu=\left(\nu^{p}\right)_{p \in \mathbb{Z}}$ with $\theta^{p} \in H\left(X^{p}, X^{p-1}\right)$ and $\nu^{p} \in P_{h}\left(X^{p}\right)$ for all $p \in \mathbb{Z}$ such that

$$
\alpha^{p-1} \theta^{p}+\theta^{p+1} \alpha^{p}=1^{p}-\nu^{p}
$$

for all $p \in \mathbb{Z}$.

\footnotetext{
${ }^{1}$ Note: We will continue to use the notation of operators for these functions even though we may not have linearity.
} 
We say that $\alpha$ is an essential complex in $\mathcal{X}$ if $\alpha^{p+1} \alpha^{p} \in K\left(X^{p}, X^{p+2}\right)$ for all $p \in \mathbb{Z}$, and $X^{p} \neq\{0\}$ only for a finite number of indices. The family of all essential complexes in $\mathcal{X}$ will be denoted by $\partial_{e}(\mathcal{X})$. We will also define $\partial_{c}(\mathcal{X})$ as $\partial_{e}(\mathcal{X}) \cap \partial(\mathcal{X})$.

It is clear that $\alpha=\left(\alpha^{p}\right)_{p \in \mathbb{Z}}$ is an element of $\partial_{c}(\mathcal{X})$ if and only if $\alpha$ is a complex of finite length in $X$, with the domain of $\alpha^{p}$ a closed subspace of $\mathcal{X}$ for all $p \in \mathbb{Z}$. This leads us to the following characterizations of Fredholm complexes.

Theorem 3. A complex $\alpha \in \partial_{c}(\mathcal{X})$ is Fredholm if and only if $\alpha$ has a homogeneous splitting.

Proof. Necessity (Adapted from the proof of Theorem II.3.14 in [1]): Assume first that $\alpha$ is Fredholm. Hence $\operatorname{dim} H^{p}(\alpha)<\infty$ for all $p \in \mathbb{Z}$. We fix an index $p$. Since $R\left(\alpha^{p-1}\right)$ is the range of a closed operator and has finite codimension is $N\left(\alpha^{p}\right)$, then it is closed and we can choose a linear projection $\pi_{1}^{p}$ of $N\left(\alpha^{p}\right)$ onto $R\left(\alpha^{p-1}\right)$. Let also $\pi_{2}^{p}$ be a homogeneous projection of $X^{p}:=D\left(\alpha^{p}\right)$ onto $N\left(\alpha^{p}\right)$. Then $\pi^{p}=\pi_{1}^{p} \pi_{2}^{p}$ is a homogeneous projection of $X^{p}$ onto $R\left(\alpha^{p-1}\right)$. Let $c^{p}: X^{p} \rightarrow X^{p} / N\left(\alpha^{p}\right)$ be the canonical projection, and let $\rho^{p}: X^{p} / N\left(\alpha^{p}\right) \rightarrow X^{p}$ be the homogeneous lifting associated with $\pi_{2}^{p}$. In other words, $\pi_{2}^{p}=1^{p}-\rho^{p} c^{p}$, where $1^{p}$ is the identity of $X^{p}$. We define a mapping $\theta^{p} \in H\left(X^{p}, X^{p-1}\right)$ in the following way.

Let $\alpha_{0}^{p-1}: X^{p-1} / N\left(\alpha^{p-1}\right) \rightarrow R\left(\alpha^{p-1}\right)$ be the bijective operator induced by $\alpha^{p-1}$. We set

$$
\theta^{p}:=\rho^{p-1}\left(\alpha_{0}^{p-1}\right)^{-1} \pi^{p} \in H\left(X^{p}, X^{p-1}\right) .
$$

We shall show that the mapping $\theta^{p}$ satisfy (1) for appropriate $\nu^{p}$. Indeed, let $x \in X^{p}$ be given. Then we have:

$$
\begin{gathered}
\theta^{p+1} \alpha^{p}(x)=\left(\rho^{p}\left(\alpha_{0}^{p}\right)^{-1} \pi^{p+1}\right)\left(\alpha^{p} x\right) \\
=\rho^{p}\left(\alpha_{0}^{p}\right)^{-1}\left(\alpha^{p} x\right)=\rho^{p} c^{p}(x)=x-\pi_{2}^{p}(x) .
\end{gathered}
$$

Since $\pi^{p}(x) \in R\left(\alpha^{p-1}\right)$, and so $\pi^{p}(x)=\alpha^{p-1}(v)$ for some $v \in X^{p-1}$, we also have:

$$
\begin{gathered}
\alpha^{p-1} \theta^{p}(x)=\alpha^{p-1} \rho^{p-1}\left(\alpha_{0}^{p-1}\right)^{-1} \pi^{p}(x) \\
=\alpha^{p-1} \rho^{p-1}\left(\alpha_{0}^{p-1}\right)^{-1}\left(\alpha^{p-1}(v)\right)=\alpha^{p-1} \rho^{p-1} c^{p-1}(v) \\
=\alpha^{p-1}\left(v-\pi_{2}^{p-1}(v)\right)=\alpha^{p-1} v=\pi^{p}(x) .
\end{gathered}
$$

Therefore

$$
\theta^{p+1} \alpha^{p}(x)+\alpha^{p-1} \theta^{p}(x)=x-\pi_{2}^{p}(x)+\pi^{p}(x),
$$

and

$$
\nu^{p}:=\pi_{2}^{p}-\pi=\left(\left.1^{p}\right|_{N\left(\alpha^{p}\right)}-\pi_{1}^{p}\right) \pi_{2}^{p}
$$

is inessential, since the linear operator induced by $\left.1^{p}\right|_{N\left(\alpha^{p}\right)}-\pi_{1}^{p}$ is a finite rank projection. Hence $\alpha$ has a homogeneous splitting. 
Sufficiency: Assume that $\alpha$ has a homogeneous splitting. Thus for each $p \in \mathbb{Z}$ we can find $\theta^{p} \in H\left(X^{p}, X^{p-1}\right)$ and $\nu^{p} \in P_{h}\left(X^{p}, X^{p}\right)$, where, as above, $X^{p}=D\left(\alpha^{p}\right)$, such that

$$
\theta^{p+1} \alpha^{p}+\alpha^{p-1} \theta^{p}=1^{p}-\nu^{p}
$$

Let $p$ be fixed.

Since $\alpha^{p-1} \theta^{p} N\left(\alpha^{p}\right) \subseteq R\left(\alpha^{p-1}\right) \subseteq N\left(\alpha^{p}\right)$, we can consider $\alpha^{p-1} \theta^{p}$ as a homogeneous operator on $N\left(\alpha^{p}\right)$. Also, on $N\left(\alpha^{p}\right)$, we have that $\alpha^{p-1} \theta^{p}=1^{p}-\nu^{p}$. And so $\nu^{p}$ can also be considered as a homogeneous operator on $N\left(\alpha^{p}\right)$. Since $N\left(\alpha^{p}\right)$ is a closed subspace of $X^{p}$, the hypothesis of lemma 2 are satisfied with $\nu^{p}$ restricted to $N\left(\alpha^{p}\right)$. Hence, $\nu^{p} \in P_{h}\left(N\left(\alpha^{p}\right), N\left(\alpha^{p}\right)\right)$.

By the definition of a homogeneous inessential operator, we know that there exists a homogeneous operator, $U^{p}$, and a compact homogeneous operator, $K^{p}$, such that $\left(1_{N\left(\alpha^{p}\right)}-\nu^{p}\right) U^{p}=1_{N\left(\alpha^{p}\right)}-K^{p}$. So,

$$
\alpha^{p-1} \theta^{p} U^{p}=1_{N\left(\alpha^{p}\right)}-K^{p} .
$$

We will use this identity to prove by contradiction that $\operatorname{dim} \frac{N\left(\alpha^{p}\right)}{R\left(\alpha^{p-1}\right)}<\infty$.

Assume that there exists an orthonormal sequence $\left(x_{n}\right)_{n=1}^{\infty}$ in $N\left(\alpha^{p}\right)$ which is orthogonal to $\alpha^{p-1} \theta^{p} N\left(\alpha^{p}\right)$. Since $K^{p}$ is compact, $\left(K^{p} x_{n}\right)$ must have a convergent subsequence. So we can assume without any loss of generality that $K^{p} x_{n} \rightarrow 0$. However, since $x_{n}$ is orthogonal to $\alpha^{p-1} \theta^{p} U^{p} x_{n}$, we have a contradictions.

Therefore, $\operatorname{dim} H^{p}(\alpha)$ is finite and since $p$ was arbitrary, the complex $\alpha$ must be Fredholm.

By defining the following functor, we get a further characterization of Fredholm complexes in terms of inessential operators.

Definition 4. If $Z, X, X_{1}, X_{2}$ are Banach spaces, we set

$$
\gamma_{Z}(X):=H(Z, X) / P_{h}(Z, X) .
$$

For every $S \in B\left(X_{1}, X_{2}\right)$, we define $\gamma_{Z}(S) \in B\left(\gamma_{Z}\left(X_{1}\right), \gamma_{Z}\left(X_{2}\right)\right)$ by the formula

$$
\gamma_{Z}(S)\left(\theta+P_{h}\left(Z, X_{1}\right)\right):=S \theta+P_{h}\left(Z, X_{2}\right)
$$

for all $\theta \in H\left(Z, X_{1}\right)$ (clearly, $S P_{h}\left(Z, X_{1}\right) \subset P_{h}\left(Z, X_{2}\right)$ ).

If $\alpha \in \partial_{e}(\mathcal{X})$, then $\gamma_{Z}(\alpha):=\left(\gamma_{Z}\left(\alpha^{p}\right)\right)_{p \in \mathbb{Z}}$.

Lemma 4. Let $\alpha, \beta \in \partial_{e}(\mathcal{X})$ be such that $\alpha^{p} \in B\left(X^{p}, X^{p+1}\right), \beta^{p} \in$ $B\left(X^{p}, X^{p+1}\right)$ and $\alpha^{p}-\beta^{p} \in P\left(X^{p}, X^{p+1}\right)$ for all $p \in \mathbb{Z}$. Then $\gamma_{Z}(\alpha)=\gamma_{Z}(\beta)$ for each Banach space $Z$.

Proof. Let $\theta \in H\left(Z, X^{p}\right)$. Then

$$
\begin{gathered}
\gamma_{Z}\left(\alpha^{p}\right)\left(\theta+P_{h}\left(Z, X^{p}\right)=\alpha^{p} \theta+P_{h}\left(Z, X^{p+1}\right)=\left(\alpha^{p}-\tilde{\alpha}^{p}\right) \theta+\tilde{\alpha}^{p} \theta+P_{h}\left(Z, X^{p+1}\right)\right. \\
=\tilde{\alpha}^{p} \theta+P_{h}\left(Z, X^{p+1}\right)=\gamma_{Z}\left(\tilde{\alpha}^{p}\right)\left(\theta+P_{h}\left(Z, X^{p}\right)\right) .
\end{gathered}
$$


Therefore, $\gamma_{Z}\left(\alpha^{p}\right)=\gamma_{Z}\left(\tilde{\alpha}^{p}\right)$ for all $p \in \mathbb{Z}$. So the two complexes are the same.

Theorem 5. Let $\alpha=\left(\alpha^{p}\right)_{p \in \mathbb{Z}} \in \partial_{e}(\mathcal{X})$. The complex $\gamma_{Z}(\alpha)$ is exact for each Banach space $Z$ if and only if $\alpha$ has a homogeneous splitting.

Proof. " $\Leftarrow "$ Assume there exists $\theta=\left(\theta^{p}\right)_{p \in \mathbb{Z}}$ and $\nu=\left(\nu^{p}\right)_{p \in \mathbb{Z}}$ with $\theta^{p} \in$ $H\left(X^{p}, X^{p-1}\right)$ and $\nu^{p} \in P_{h}\left(X^{p}\right)$ for all $p \in \mathbb{Z}$ such that

$$
\alpha^{p-1} \theta^{p}+\theta^{p+1} \alpha^{p}=1^{p}-\nu^{p}, \quad \forall p \in \mathbb{Z} .
$$

For each $p$, let $\varphi^{p} \in H\left(Z, X^{p}\right)$ be such that $\alpha^{p} \varphi^{p} \in P_{h}\left(Z, X^{p+1}\right)$. Then

$$
\begin{gathered}
\left(\alpha^{p-1} \theta^{p}+\theta^{p+1} \alpha^{p}\right)\left(\varphi^{p}\right)=\left(1^{p}-\nu^{p}\right)\left(\varphi^{p}\right), \\
\varphi^{p}=\alpha^{p-1}\left(\theta^{p} \varphi^{p}\right)+\left(\theta^{p+1} \alpha^{p} \varphi^{p}+\nu^{p} \varphi^{p}\right) \in \alpha^{p-1}\left(\theta^{p} \varphi^{p}\right)+P_{h}\left(Z, X^{p}\right) .
\end{gathered}
$$

Thus, $\varphi^{p}+P_{h}\left(Z, X^{p}\right) \in R\left(\gamma_{Z}\left(\alpha^{p-1}\right)\right)$. Therefore, the complex $\gamma_{Z}(\alpha)$ is exact.

" $\Rightarrow$ " Assume that $\gamma_{Z}(\alpha)$ is exact. If $X^{p}=D\left(\alpha^{p}\right)$ is a closed subspace of $X$, we may assume, $X^{p}=\{0\}$ for all $p<0$.

Let also $n \geq 0$ be the least integer with the property $X^{p}=\{0\}$ for all $p>n$. We shall show that for each $0 \leq p \leq n$, we can find operators $\theta^{p} \in H\left(X^{p}, X^{p-1}\right)$ and $\nu^{p} \in P_{h}\left(X^{p}\right)$ which satisfy $(1)$.

If $p=n$, from the exactness of the complex $\gamma_{Z}(\alpha)$ for $Z:=X^{n}$, we obtain the existence of an operator $\theta^{n} \in H\left(X^{n}, X^{n-1}\right)$ such that $\alpha^{n-1} \theta^{n}-1^{n} \in P_{h}\left(X^{n}\right)$. Then we set $\nu^{n}:=1^{n}-\alpha^{n-1} \theta^{n}$.

Assume that we have found mappings $\theta^{q}, \nu^{q}$ for all $q \geq p, q \leq n$. Note that

$$
\begin{gathered}
\alpha^{p-1}\left(1^{p-1}-\theta^{p} \alpha^{p-1}\right)=\alpha^{p-1}-\left(1^{p}-\nu^{p}-\theta^{p+1} \alpha^{p}\right) \alpha^{p-1} \\
=\nu^{p} \alpha^{p-1}+\theta^{p+1} \alpha^{p} \alpha^{p-1} \in P_{h}\left(X^{p}\right),
\end{gathered}
$$

in virtue of (1). From the exactness of the complex $\gamma_{Z}(\alpha)$ for $Z:=X^{p-1}$, we deduce the existence of an operator $\theta^{p-1} \in H\left(X^{p-1}, X^{p-2}\right)$ such that

$$
\alpha^{p-2} \theta^{p-1}=1^{p-1}-\theta^{p} \alpha^{p-1}-\nu^{p-1},
$$

where $\nu^{p-1} \in P_{h}\left(X^{p-1}\right)$. Thus $\alpha$ has a homogeneous splitting.

Let's put all of these results together in the following corollary.

Corollary 6. The following are equivalent for a complex $\alpha \in \partial_{c}(\mathcal{X})$.

(a) $\alpha$ is Fredholm

(b) $\alpha$ has a homogeneous splitting

(c) The complex $\gamma_{Z}(\alpha)$ is exact for each Banach space $Z$.

Theorem 7. Let

$$
0 \rightarrow X^{0} \stackrel{\alpha^{0}}{\longrightarrow} X^{1} \stackrel{\alpha^{1}}{\longrightarrow} \cdots \stackrel{\alpha^{n-1}}{\longrightarrow} X^{n} \rightarrow 0
$$

be a Fredholm complex of Banach spaces and continuous operators. If

$$
0 \rightarrow X^{0} \stackrel{\tilde{\alpha}^{0}}{\longrightarrow} X^{1} \stackrel{\tilde{\alpha}^{1}}{\longrightarrow} \cdots \stackrel{\tilde{\alpha}^{n-1}}{\longrightarrow} X^{n} \rightarrow 0
$$


is another complex such that $\alpha^{p}-\tilde{\alpha}^{p} \in P\left(X^{p}, X^{p+1}\right)$ for all $p=0,1, \ldots, n$, then the latter complex is also Fredholm.

Proof. Since the complexes $\gamma_{Z}(\alpha)$ and $\gamma_{Z}(\tilde{\alpha})$ are the same, we have that if one is Fredholm, then so is the other.

\section{REFERENCES}

1. C.-G. Ambrozie and F.-H. Vasilescu, Banach space complexes. Kluwer Academic Publishers, Dordrecht, 1995.

2. R. G. Bartle and L. M. Graves, Mappings between function spaces. Trans. Amer. Math. Soc. 72(1952), 400-413.

3. S. R. Caradus, W. E. Pfaffenberger, and Bertram Yood, Calkin algebras and algebras of operators on Banach spaces. Marcel Dekker, Inc., New York, 1974.

4. R. G. Douglas, Banach algebra techniques in operator theory. Springer-Verlag New York Inc., New York, 1998.

5. I. C. Gohberg, A. S. Markus, and I. A. Feldman, Normally solvable operators and ideals associated with them. Amer. Math. Soc. Transl. II Ser. 61, 63-84, 1967; Russan original: Izv. Mold. Fil. Akad. Nauk SSSR 10(76)(1960), 51-70.

6. A. Pietsch, Operator ideals. North-Holland Publishing Company, Amsterdam, New York, Oxford, 1980.

(Received 20.09.2000)

Author's address:

University of California

Santa Barbara, CA 93106

USA

E-mail: gleason@math.ucsb.edu 